\title{
Fourth-order explicit hybrid method for solving special second-order ordinary differential equations
}

\begin{abstract}
In this paper, a fourth-order explicit hybrid method with constant step-size is developed. The free parameters are chosen so that the error constant is small. Numerical comparisons are carried out to show the advantage of the new method for solving special second order ordinary differential equations.
\end{abstract}

Keyword: Error constant; Hybrid method; Second order ordinary differential equations 